\title{
Effect of radiation-like solid on small-scale CMB anisotropies
}

\author{
Peter Mészáros and Vladimír Balek \\ Department of Theoretical Physics, Comenius University, Bratislava, Slovakia
}

July 12, 2017

\begin{abstract}
We compute the CMB angular power spectrum in the presence of a radiation-like solid elastic matter with the same pressure to energy density ratio as radiation but with nonzero shear modulus. For the values of shear modulus that are close enough to zero, so that the effect of the solid on large-scale anisotropies remains within cosmic variance, we find that there is an observable effect of the solid on small-scale anisotropies.
\end{abstract}

\section{Introduction}

The idea of solid matter was introduced into cosmology in an attempt to give an alternative explanation of the acceleration of the universe, see [1] where the dark energy was replaced by a solid with negative pressure to energy density ratio $w$. Further development of the theory $2,3,4,4,6,6,8,8$ included works where inflation was driven by a solid (solid inflation) [9, 10, 11, 12, 13. An important example of how such solid can materialize are cosmic strings and domain walls [2, 3, 8.

The effect of a solid can be obtained by any mechanism leading to nondiagonal 3-space components of energy-momentum tensor of the desired form. In particular, one can replace internal coordinates of the solid by three scalar fields $\phi^{A}(x)$ and consider a Lagrangian $\mathcal{L}[\phi]$ depending on them [10, 11].

To extend the parameter space of the theory one can consider also a solid with positive pressure to energy density ratio [14. An important special case is the radiation-like solid with $w=1 / 3$, which does not influence the evolution of the unperturbed universe if the total energy density of radiation and solid equals the energy density of radiation in the standard case. Materialization of such a solid could be a Coulomb crystal with relativistic Fermi gas of moving particles, or a network of speculative 'spring-like' strings with energy inversely proportional to their length.

Radiation-like solid, like any matter that has $w \geq-1 / 3$, produces decelerating expansion of the universe. Thus, it cannot be regarded as an alternative to dark energy. The reason why we are studying it is that it would, if present in the universe, modify the evolution of perturbations and leave an imprint on CMB anisotropies.

The solid component of matter considered in cosmology differs greatly from the solids known from everyday experience, since it has to be stretched by many orders of magnitude in the course of expansion of the universe, and still remain solid. Such behavior is necessary if we want to preserve the cosmological principle. If the solid was motionless while the other components of cosmic matter were expanding, different observers would see it in different states of motion, hence the universe would look differently for them. 
Solid matter affects the evolution of perturbations if it appears with flat internal geometry and nonzero shear stress acting in it. Such solidification cannot occur in pure radiation and must be related to some other kind of particles distributed anisotropically before solidification. The particles could possibly appear in the universe as a remnant of solid inflation.

However well the current observations are explained by standard theory with radiation, baryonic matter, cold dark matter and dark energy, future observations could be nevertheless lacking in full explanation within the standard theory and some nonstandard theories, including the model presented in this work, might become relevant.

The effect of a radiation-like solid on long-wavelength perturbations and on the coefficients of angular power spectrum of cosmic microwave background (CMB) with very low multipole moments was studied in [15]. In the long-wavelength limit the sound speed does not appear in the equations for perturbations (see (7.69) in [17]), therefore one can suppose the cold dark matter $(\mathrm{CDM})$ to be coupled to the baryon-radiation plasma. Also, considering neutrinos to be coupled to photons should not change the results qualitatively.

In this work we study how a radiation-like solid appearing in the universe shortly after inflation influences the CMB angular power spectrum for arbitrary multipole moments. To get the high moments right, one must consider a model in which the CDM and neutrinos are decoupled from the baryon-radiation plasma. Rather than merely extending the existing theory of a universe filled with ideal fluid, which uses gauge invariant variables as basic objects [16, 17, we build the theory from the scratch in the proper-time comoving gauge [18. In section 2 we derive equations governing the evolution of perturbations in this gauge, in section 3 we enumerate important effects contributing to the CMB anisotropies, in section 4 we summarize the effects of the radiation-like solid on the CMB anisotropies and in the last section we discuss results. To complete the analysis, in the appendix we solve equations from section 2 analytically in the long-wavelength limit. We use the signature of the metric tensor $(+---)$ and the units in which $c=16 \pi G=1$.

\section{Equations for perturbations}

Observations of the CMB anisotropies can be explained by the perturbation theory and of the three kinds of perturbations, scalar, vector and tensor, scalar perturbations appear to give the dominant contribution to the observed anisotropies. We will consider scalar perturbation theory in a flat Friedmann-Robertson-Walker-Lemaître (FRWL) universe filled with radiation, baryonic matter and $\mathrm{CDM}$, which appears to be a realistic model of the universe in the pre-recombination era. However, we will add an elastic radiation-like matter with nonzero shear modulus to the other matter components. The presence of the radiationlike solid, which has the pressure to energy density ratio $w=1 / 3$ just like radiation (photons and, in the early universe, neutrinos), does not change the evolution of the unperturbed universe provided that the total energy density of all radiation-like components is unchanged.

We will use proper-time comoving gauge [18] in which the (00)-component of the metric tensor is unperturbed and the shift vector $\delta \mathbf{x}$ is zero for radiation-like solid as well as for all kinds of matter coupled to it. In this gauge the scalar part of metric takes the form

$$
d s^{(S) 2}=a^{2}\left\{d \eta^{2}+2 B_{, i} d \eta d x^{i}-\left[(1-2 \psi) \delta_{i j}-2 E_{, i j}\right] d x^{i} d x^{j}\right\}
$$

where $a$ is the scale parameter, $\eta$ is the conformal time and $B, \psi$ and $E$ are the functions describing scalar perturbations. If the only matter coupled to the radiation-like solid is the baryon-radiation plasma, the scalar part of energy-momentum tensor is

$$
\begin{aligned}
& T_{0}{ }^{0}=\rho+\rho_{+}^{b s \gamma}(3 \psi+\mathcal{E})+\delta \rho^{d}+\delta \rho^{\nu}, \quad T_{i}{ }^{0(S)}=\rho_{+}^{b s \gamma} B_{, i}+\rho^{d} \vartheta_{, i}+\rho_{+}^{\nu} \sigma_{, i}, \\
& T_{i}{ }^{j(S)}=-p \delta_{i}^{j}-K(3 \psi+\mathcal{E}) \delta_{i}^{j}-2 \mu E_{, i j}^{T}-2 \mathcal{N} a^{-1} E_{, \eta i j}^{T}-(1 / 3) \delta \rho^{\nu} \delta_{i}^{j},
\end{aligned}
$$


where $\rho$ is the total energy density, $\rho_{+}=\rho+p, p$ being pressure, $\rho^{d}$ is the energy density of the CDM, $\rho^{\nu}$ is the energy density of neutrinos, $\rho^{b s \gamma}$ is the energy density of the system of baryons, solid and photons, $\delta \rho^{d}$ and $\delta \rho^{\nu}$ are perturbations of the energy density of CDM and neutrinos, $\vartheta_{, i}$ and $\sigma_{, i}$ are scalar parts (longitudinal parts in Helmholtz decomposition) of the velocity of CDM and neutrinos with respect to other matter, $K=(1 / 3) \rho_{+}^{s \gamma}$ is the compressional modulus of the system of solid and photons, $\mu$ is the shear modulus of the radiation-like solid, $\mathcal{N}$ is the shear viscosity coefficient due to the coupling of photons and baryonic matter before recombination (which is responsible for Silk damping), $\mathcal{E}=\triangle E$ and $E_{, i j}^{T} \equiv E_{, i j}-(1 / 3) \triangle E \delta_{i j}$ is the traceless part of the tensor $E_{, i j}$.

Energy-momentum tensor with nondiagonal spatial components can be obtained from any given Lagrangian $\mathcal{L}[\phi]$ which is invariant under internal rotations and translations [10, 11],

$$
\begin{array}{ll}
\phi^{A} \rightarrow M_{B}^{A} \phi^{B}, & \phi^{A} \rightarrow \phi^{A}+C^{A}, \\
M_{B}^{A} \in S O(3), & C^{A} \in \mathbb{R}^{3}, \quad A, B=1,2,3,
\end{array}
$$

where the capital indices are raised and lowered by the Euclidean metric $\delta_{A B}$. If the object described by the theory is solid matter, the three-component field $\phi^{A}$ is interpreted as the socalled spatial internal coordinates which move with the matter. As a result, in the perturbed FRWL universe we have

$$
\phi^{A}(x)=\delta_{i}^{A} x^{i}-\delta x^{A}(x)
$$

where the fields $\delta x^{A}$ describing the perturbed state of the solid play the role of Goldstone boson fields in the field formulation of the theory. The Lagrangian leading to the energymomentum tensor (2) up to the first order of perturbation theory, except for the Silk damping term, is 14

$$
\mathcal{L}=\mathcal{L}_{0}+c_{1} \partial_{\mu} \phi^{A} \partial^{\mu} \phi_{A}+\left(c_{2} \delta_{A B} \delta_{C D}+c_{3} \delta_{A C} \delta_{B D}\right) \partial_{\mu} \phi^{A} \partial^{\mu} \phi^{B} \partial_{\nu} \phi^{C} \partial^{\nu} \phi^{D},
$$

where $\mathcal{L}_{0}=\rho+9\left(K-\rho_{+}\right) / 8, c_{1}=\left(3 K-\rho_{+}\right) a^{2} / 4, c_{2}=\left(\lambda+\rho_{+}\right) a^{4} / 8, \lambda$ being the first Lame coefficient related to compressional modulus by the formula $K=\lambda+2 \mu / 3$, and $c_{3}=$ $\left(\mu-\rho_{+}\right) a^{4} / 4$. The Silk damping term is not obtained from the Lagrangian, but computed directly from the expression of energy-momentum tensor for an imperfect fluid [17.

For the variables $\psi, \mathcal{E}, B, \vartheta, \delta \rho^{d}, \sigma$ and $\delta \rho^{\nu}$ we have equations

$$
\begin{aligned}
& 4 \psi^{\prime}=a^{2}\left(\rho_{+}^{b s \gamma} B+\rho^{d} \vartheta+\rho^{\nu} \sigma\right), \\
& 4 \triangle(\mathcal{H} B+\psi)-4 \mathcal{H}(3 \psi+\mathcal{E})^{\prime}=a^{2}\left(\rho_{+}^{b s \gamma}(3 \psi+\mathcal{E})+\delta \rho^{d}+\delta \rho^{\nu}\right), \\
& \rho_{+}^{b s \gamma} B^{\prime}=\mathcal{H}\left(3 K-\rho_{+}^{b s \gamma}\right) B+3 K \psi+(K+4 \mu / 3) \mathcal{E}+4 \mathcal{N} a^{-1} \mathcal{E}^{\prime} / 3, \\
& \vartheta^{\prime}=-\mathcal{H} \vartheta \\
& \left(\delta \rho^{d} / \rho^{d}\right)^{\prime}-(3 \psi+\mathcal{E})^{\prime}=\triangle(\vartheta-B), \\
& 4 \sigma^{\prime}=\delta \rho^{\nu} / \rho^{\nu} \\
& 3\left(\delta \rho^{\nu} / \rho^{\nu}\right)^{\prime}-4(3 \psi+\mathcal{E})^{\prime}=4 \triangle(\vartheta-B),
\end{aligned}
$$

where the prime denotes differentiation with respect to $\eta$ and $\mathcal{H}=a^{\prime} / a$. Equations (6) and (7) are derived from $\left(\begin{array}{l}0 \\ i\end{array}\right)$ and $\left(\begin{array}{l}0 \\ 0\end{array}\right)$ components of Einstein field equations, $2 G_{i}{ }^{0}=T_{i}{ }^{0}$ and $2 G_{0}{ }^{0}=T_{0}{ }^{0}$, equations (8), (9) and (11) are obtained from the momentum conservation law, $T_{i ; \mu}^{\mu}=0$, written for the system of baryon-radiation plasma and radiation-like solid, CDM and neutrinos respectively, and equations (10) and (12) follow from the energy conservation law, $T_{0}{ }^{\mu} ; \mu=0$, written for CDM and neutrinos. All equations are obtained in the first order of the perturbation theory in the case when the internal geometry of the radiation-like solid is flat.

Equations (6)-(12) are not invariant under coordinate transformations because the propertime comoving gauge allows for a residual transformation $\eta \rightarrow \eta+a^{-1} \delta t(\mathbf{x})$, where $\delta t(\mathbf{x})$ is 
the local shift of the moment at which the time count starts. The function $\mathcal{E}$ is invariant under this transformation and $B, \psi, \mathrm{CDM}$ energy density contrast $\delta^{d}=\delta \rho^{d} / \rho^{d}$, neutrino energy density contrast $\delta^{\nu}=\delta \rho^{\nu} / \rho^{\nu}, \vartheta$ and $\sigma$ can be rewritten as

$$
\begin{aligned}
& B=\mathcal{B}+\chi, \quad \psi=-\mathcal{H} \chi, \\
& \delta^{d}=\hat{\delta}^{d}-3 \mathcal{H} \chi, \quad \vartheta=\hat{\vartheta}+\chi, \\
& \delta^{\nu}=\hat{\delta}^{\nu}-4 \mathcal{H} \chi, \quad \sigma=\hat{\sigma}+\chi,
\end{aligned}
$$

where $\chi$ transforms as $\chi \rightarrow \chi+a^{-1} \delta t(\mathbf{x})$ and $\mathcal{B}, \hat{\delta}^{d}, \hat{\delta}^{\nu}, \hat{\vartheta}$ and $\hat{\sigma}$ are invariant. It is convenient to introduce a rescaled time $\zeta=\eta / \eta_{*}$, where $\eta_{*}=\eta_{e q} /(\sqrt{2}-1)$ and $\eta_{e q}$ denotes the moment when the energy density of matter is equal to the energy density of radiation. As long as the contribution of dark energy to the total energy density is negligible, which is surely the case before recombination, the scale parameter can be written as $a=a_{e q} \zeta(\zeta+2)$.

Using (6) and (13) and considering perturbations of the form of a plane wave with the comoving wave vector $\mathbf{k}$, equations (17)-(12) can be rewritten into the invariant form

$$
\begin{aligned}
& \mathcal{E}^{\prime}=-\left(s^{2}+3 \alpha_{b s \gamma} \tilde{\mathcal{H}}^{2}\right) \tilde{\mathcal{B}}-\alpha_{b s \gamma} \tilde{\mathcal{H}} \mathcal{E}-3 \alpha_{d} \tilde{\mathcal{H}}^{2} \Theta-\alpha_{d} \tilde{\mathcal{H}} \hat{\delta}^{d}-3 \alpha_{\nu} \tilde{\mathcal{H}}^{2} \Sigma-\frac{3}{4} \alpha_{\nu} \tilde{\mathcal{H}}^{\nu} \\
& \tilde{\mathcal{B}}^{\prime}=\left(3 c_{s 0}^{2}+\alpha_{b s \gamma}-1\right) \tilde{\mathcal{H}} \tilde{\mathcal{B}}+c_{s \| \mid}^{2} \mathcal{E}+\Xi \mathcal{E}^{\prime}+\alpha_{d} \tilde{\mathcal{H}} \Theta+\alpha_{\nu} \tilde{\mathcal{H}} \Sigma \\
& \Theta^{\prime}=\alpha_{b s \gamma} \tilde{\mathcal{H}} \tilde{\mathcal{B}}+\left(\alpha_{d}-1\right) \tilde{\mathcal{H}} \Theta+\alpha_{\nu} \tilde{\mathcal{H}} \Sigma \\
& \hat{\delta}^{d \prime}=\mathcal{E}^{\prime}+s^{2}(\tilde{\mathcal{B}}-\Theta) \\
& \Sigma^{\prime}=\alpha_{b s \gamma} \tilde{\mathcal{H}} \tilde{\mathcal{B}}+\alpha_{d} \tilde{\mathcal{H}} \Theta+\alpha_{\nu} \tilde{\mathcal{H}} \Sigma+\frac{1}{4} \hat{\delta}^{\nu} \\
& \hat{\delta}^{\nu \prime}=\frac{4}{3} \mathcal{E}^{\prime}+\frac{4}{3} s^{2}(\tilde{\mathcal{B}}-\Sigma)
\end{aligned}
$$

where the prime denotes differentiation with respect to $\zeta, s=k \eta_{*}, \tilde{\mathcal{H}}=a^{\prime} / a$ with the redefined prime, $\tilde{\mathcal{B}}=\mathcal{B} / \eta_{*}, \Theta=\hat{\vartheta} / \eta_{*}, \Sigma=\hat{\sigma} / \eta_{*}, \alpha_{b s \gamma}=3 \rho_{+}^{b s \gamma} /(2 \rho), \alpha_{d}=3 \rho^{d} /(2 \rho)$, $\alpha_{\nu}=3 \rho_{+}^{\nu} /(2 \rho)$ and $\Xi=4 \mathcal{N} /\left(3 a \rho_{+}^{b s \gamma} \eta_{*}\right)$. The two sound speeds appearing in equation (15), auxiliary sound speed $c_{s 0}$ and longitudinal sound speed of the baryon-radiation plasma with radiation-like solid coupled to it $c_{s \|}$, are defined as

$$
c_{s 0}^{2}=\frac{K}{\rho_{+}^{b s \gamma}}, \quad c_{s \|}^{2}=c_{s 0}^{2}+\frac{4}{3} \frac{\mu}{\rho_{+}^{b s \gamma}}=(1+3 \xi) c_{s 0}^{2},
$$

where $\xi$ is the dimensionless shear modulus defined as

$$
\xi=\frac{\mu}{\rho^{s \gamma}},
$$

$\rho^{s \gamma}$ being the sum of energy densities of solid and photons. The shear viscosity coefficient $\mathcal{N}$ entering equation (15) is proportional to the mean free time for photon scattering, which is in turn inversely proportional to electron ionization fraction. In our computation, we took into account that this fraction drops smoothly from 1 to a value close to zero during recombination (see $§ 3.6 .2$ and $\S 3.6 .3$ in [17]).

Equations (14)-(19) describe completely the evolution of perturbations in the presence of radiation-like solid. They generalize equations (4) in [15] valid in long-wavelength limit only.

Perturbations generated during inflation are long-wavelength (superhorizon) afterwards, therefore the initial conditions for equations describing their evolution, set at some moment $\zeta_{\text {inf }}$ shortly after the end of inflation, are $\mathcal{E}\left(\zeta_{\text {inf }}\right)=(-9 / 2) \Phi^{(0)}, \tilde{\mathcal{B}}\left(\zeta_{\text {inf }}\right)=0, \Theta\left(\zeta_{\text {inf }}\right)=$ $0, \hat{\delta}^{d}\left(\zeta_{\text {inf }}\right)=(-9 / 2) \Phi^{(0)}, \Sigma\left(\zeta_{\text {inf }}\right)=0, \hat{\delta}^{\nu}\left(\zeta_{\text {inf }}\right)=-6 \Phi^{(0)}$, where $\Phi^{(0)}$ is the primordial Newtonian potential. Suppose the time of solidification $\zeta_{s}$ (the time when the radiation-like solid was formed) was greater than the time $\zeta_{\text {inf }}$ but much smaller than the time $\zeta_{e q}$ (the time 
of matter-radiation equality). Then, the initial conditions at some moment $\zeta_{\text {in }}>\zeta_{s}$, when the numerical integration of the equations for perturbations starts, can be obtained by solving the equations between the times $\zeta_{\text {inf }}$ and $\zeta_{\text {in }}$ analytically, neglecting the contribution of the CDM and baryonic matter to the energy density and considering the primordial perturbations to be superhorizon up to the moment $\zeta_{\text {in }}$. In this way we find

$$
\begin{aligned}
\mathcal{E}\left(\zeta_{i n}\right) & =-\frac{9}{4 n}\left(M \hat{\zeta}^{-m}-m \hat{\zeta}^{-M}\right) \\
\tilde{\mathcal{B}}\left(\zeta_{i n}\right) & =\frac{9 \zeta_{s}}{24 n}\left[M(M+1) \hat{\zeta}^{M}-m(m+1) \hat{\zeta}^{m}\right], \\
\Theta\left(\zeta_{\text {in }}\right) & =\tilde{\mathcal{B}}\left(\zeta_{\text {in }}\right)+\frac{9}{4 n} \xi \zeta_{s}\left(\hat{\zeta}^{M}-\hat{\zeta}^{m}\right), \\
\hat{\delta}^{d}\left(\zeta_{\text {in }}\right) & =\mathcal{E}\left(\zeta_{\text {in }}\right), \\
\Sigma\left(\zeta_{\text {in }}\right) & =\tilde{\mathcal{B}}\left(\zeta_{\text {in }}\right)-\frac{9}{2} \xi \zeta_{\text {in }}, \\
\hat{\delta}^{\nu}\left(\zeta_{\text {in }}\right) & =\frac{4}{3} \mathcal{E}\left(\zeta_{\text {in }}\right)-36 \xi
\end{aligned}
$$

where $\hat{\zeta}=\zeta_{\text {in }} / \zeta_{s}, n=\sqrt{1-24 \xi} / 2, m=1 / 2-n$ and $M=1 / 2+n$.

We have assumed that the moment $\zeta_{\text {inf }}$ occurred shortly, but not immediately, after the end of inflation, so that the decaying part of perturbations was negligible then. We have also assumed that the moment $\zeta_{\text {in }}$ followed soon enough after the moment $\zeta_{s}$, so that it occurred much sooner than the time of equality $\zeta_{e q}$ and the short-scale perturbations were superhorizon then. The latter assumption at the same time enabled us to use the law $a \propto \zeta$ valid for radiation-dominated universe, when deriving the conditions (22). The constraints on $\zeta_{\text {inf }}$ and $\zeta_{\text {in }}$ leave us with a wide interval of possible values of the solidification time $\zeta_{s}$. It turns out, however, that the results practically do not depend on the actual value of $\zeta_{s}$, so that we could have chosen $\zeta_{s}=10^{-9}$, which corresponds to energy about $500 \mathrm{MeV}$, in our calculations, although the solid presumably appeared at an energy scale much greater than the energies accessible at present-day accelerators.

The perturbations were supposed to be purely adiabatic at the moment $\zeta_{\text {inf }}$. If entropic perturbations emerged from inflation, the time $\zeta_{\text {inf }}$ should be shifted to the first period of local thermal equilibrium with no non-zero conserved quantities, see $\$ 5.4$ in [16]. Note that after solidification, the perturbations do not retain the simple form derived in [16] for adiabatic perturbations. For an analysis of this effect, see $\S 9$ in [10].

Instead of the proper-time comoving gauge, one often uses Newtonian gauge in which the scalar part of space-time metric is diagonal. Metric in the Newtonian gauge is given by two potentials invariant under coordinate transformations, $\Phi$, called Newtonian potential, and $\Psi$, as $g_{00}=a^{2}(1+2 \Phi)$ and $g_{i j}=-a^{2}(1-2 \Psi) \delta_{i j}$. The functions $\Phi$ and $\Psi$ can be written as [17]

$$
\Psi=\mathcal{H}\left(\mathcal{B}-E^{\prime}\right), \quad \Phi=\Psi-\mu a^{2} E
$$

where the difference between $\Phi$ and $\Psi$ is given by the traceless part of the equation $2 G_{i}{ }^{j}=T_{i}{ }^{j}$. The Silk damping is omitted here since its contribution to the second equation in (23) is negligible.

Characteristics of matter, density contrast $\delta=\delta \rho / \rho$ and "velocity potential" $\phi$ defining the scalar part of velocity according to $u_{i}^{(S)}=\phi_{, i}$, can be computed in Newtonian gauge as well. In this way we obtain invariant functions $\bar{\delta}$ and $\bar{\phi}$, which are often viewed as physical quantities. For CDM and neutrinos, the physical density contrasts and "velocity potentials" are

$$
\begin{array}{ll}
\bar{\delta}^{d}=\hat{\delta}^{d}+3 \mathcal{H}\left(\mathcal{B}-E^{\prime}\right), & \bar{\vartheta}=\hat{\vartheta}+\mathcal{B}-E^{\prime}, \\
\bar{\delta}^{\nu}=\hat{\delta}^{\nu}+4 \mathcal{H}\left(\mathcal{B}-E^{\prime}\right), & \bar{\sigma}=\hat{\sigma}+\mathcal{B}-E^{\prime} .
\end{array}
$$


Another useful relation,

$$
\overline{\delta \rho}^{b s \gamma}=\rho_{+}^{b s \gamma}(3 \Psi+\mathcal{E})
$$

is valid also for separate components of the system consisting of baryons, solid and photons.

Note that the velocity potentials $\hat{\vartheta}$ and $\hat{\sigma}$ define the spatial part of 4 -velocity with the lower index. In order to acquire physical velocities one must rise the index with respect to the perturbed metric to obtain the potentials $\mathcal{B}-\hat{\vartheta}$ for the CDM physical velocity and $\mathcal{B}-\hat{\sigma}$ for the neutrino physical velocity, both with respect to the baryon-radiation plasma. Furthermore, for the long-wave perturbations we have $\bar{\delta}^{d}=(3 / 4) \bar{\delta}^{\gamma}$ and $\bar{\delta}^{\nu}=\bar{\delta}^{\gamma}$ [19], and since $\bar{\delta}^{\gamma}=4 \Psi+4 \mathcal{E} / 3$ (see equation (25) ) and $\bar{\delta}^{d}=\hat{\delta}^{d}+3 \Psi, \bar{\delta}^{\nu}=\hat{\delta}^{\nu}+4 \Psi$ (see the first equation in (23)), we can see that the differences $\hat{\delta}^{d}-\mathcal{E}$ and $\hat{\delta}^{\nu}-(4 / 3) \mathcal{E}$ are deviations of the actual density contrasts from their limit values.

\section{Fluctuations of the CMB temperature}

The CMB anisotropies are given by perturbations around the time of last scattering and effects influencing photons during their propagation to the observer, which can be described by the relativistic Boltzmann equation for polarization-dependent distribution function of photons. The solution for temperature fluctuations $\delta T / T$ can be expressed in the form of line-of-sight integral, and if we restrict ourselves to scalar fluctuations, we can write it as a sum of two terms (see $\S 7.1$ in [16]),

$$
\frac{\delta T}{T}\left(\eta_{0}, \mathbf{l}\right)=\left(\frac{\delta T}{T}\right)_{\text {early }}\left(\eta_{0}, \mathbf{l}\right)+\left(\frac{\delta T}{T}\right)_{\mathrm{ISW}}\left(\eta_{0}, \mathbf{l}\right),
$$

where $\eta_{0}$ is the conformal time today and $\mathbf{l}$ is the unit vector pointing towards the observer from the place in the sky from which the radiation is coming. The first term represents fluctuations which appeared when there was significant amount of free electrons in the universe (this includes the period of reionization, which is however not considered here), and the second term, called integrated Sachs-Wolfe effect, represents fluctuations which arise from the action of metric perturbations on radiation during its propagation to the observer.

The early part of temperature fluctuations simplifies if we assume that photons are in local thermal equilibrium during the whole period of recombination, and that they can scatter for the last time at any moment $\eta_{l}$ with the probability distribution given by their mean free time at that moment. The line-of-sight integral then reduces to an integral over the times $\eta_{l}$, with the integrand that can be written as

$$
\left(\frac{\delta T}{T}\right)_{\text {early }}\left(\eta_{0}, \mathbf{l} ; \eta_{l}\right)=\frac{1}{4} \delta_{\gamma}\left(\eta_{l}, \mathbf{l}\right)+\Phi\left(\eta_{l}, \mathbf{l}\right)+\mathbf{v}\left(\eta_{l}, \mathbf{l}\right) \cdot \mathbf{l},
$$

where $\delta_{\gamma}=\overline{\delta \rho}^{\gamma} / \rho^{\gamma}$ is the photon density contrast in Newtonian gauge (for simplicity, we skip the bar over $\delta), \mathbf{v}$ is the local velocity of the radiating matter, and $\delta_{\gamma}(\eta, \mathbf{l}), \Phi(\eta, \mathbf{l})$ and $\mathbf{v}(\eta, \mathbf{l})$ denote the values of functions $\delta_{\gamma}, \Phi$ and $\mathbf{v}$ at the conformal time $\eta$ and at the position where the photon arriving in the direction 1 was at that time. The first term in (27) is the local contribution to $\delta T / T$ as computed from the proportionality $\rho_{\gamma} \propto T^{4}$ (StefanBoltzmann law), the second term is called Sachs-Wolfe effect and comes from the action of metric perturbations on radiation in the place of last scattering, and the third term is due to Doppler effect, which contributes to the temperature fluctuations because the radiating matter at the time of last scattering is not static. Note also that equation (25), when written for photons, implies that $\delta_{\gamma}$ can be expressed in terms of the perturbations of metric as $\delta_{\gamma}=4(\Psi+\mathcal{E} / 3)$. 
The ISW part of temperature fluctuations is known to be negligible, except for fluctuations with greatest wavelengths, in case the universe is filled with ideal fluid. A question arises whether this is the case also when the universe contains a solid component. The answer is apparently "yes", as can be seen most easily if we estimate the integral of $\Phi^{\prime}+\Psi^{\prime}$, which appears in the part of the angular power spectrum coming from $(\delta T / T)_{I S W}$, by keeping the leading terms in the expansion of $\Phi^{\prime}+\Psi^{\prime}$ in $s$ only.

The velocity $\mathbf{v}$ can be obtained from the energy conservation law for radiation, written in Newtonian gauge,

$$
3\left(\delta_{\gamma}-4 \Psi\right)^{\prime}+4 \nabla \cdot \mathbf{v}=0 .
$$

(See (7.110) in [17, where $\Phi$ is to be replaced by $\Psi$ in order that the equation stays valid in the presence of solid matter.) Considering perturbations of the form of the a plane wave with the comoving wave vector $\mathbf{k}$, we find

$$
\mathbf{v}\left(\eta_{l}, \mathbf{l}\right) \cdot \mathbf{l}=i \frac{3}{4} \frac{\mathbf{k} \cdot \mathbf{l}}{k^{2}} \frac{\partial}{\partial \eta}\left(\delta_{\gamma}-4 \Psi\right)_{\eta_{l}},
$$

so that the contribution of photons which scattered at the last time at the moment $\eta_{l}$ to the fluctuations of temperature seen in the direction $\mathbf{l}$ is approximately

$$
\frac{\delta T}{T}\left(\eta_{0}, \mathbf{l} ; \eta_{l}\right)=\int \frac{d^{3} k}{(2 \pi)^{3 / 2}}\left(\alpha(\mathbf{k})+\beta(\mathbf{k}) \partial_{0}\right)_{\eta_{l}} e^{i \mathbf{k} \cdot \mathbf{l}\left(\eta_{l}-\eta_{0}\right)}
$$

where $\partial_{0}=\partial / \partial \eta_{0}$ and $\alpha(\mathbf{k})$ and $\beta(\mathbf{k})$ are defined as

$$
\alpha(\mathbf{k})=\frac{1}{4} \delta_{\gamma \mathbf{k}}+\Phi_{\mathbf{k}}, \quad \beta(\mathbf{k})=-\frac{3}{4 k^{2}} \frac{\partial}{\partial \eta}\left(\delta_{\gamma \mathbf{k}}-4 \Psi_{\mathbf{k}}\right)
$$

The functions $\delta_{\gamma \mathbf{k}}, \Phi_{\mathbf{k}}$ and $\Psi_{\mathbf{k}}$ entering here are amplitudes of the corresponding perturbations in the form of plane waves, which depend on the conformal time $\eta$ only.

In what follows we suppose that the dimensionless shear modulus $\xi$ defined in (21) is small in absolute value. The numerical solution of equations for perturbations revealed that for $|\xi| \lesssim 5 \cdot 10^{-4}$ it is sufficient to consider the functions $\alpha$ and $\beta$ in the form of Taylor expansion up to the first order in $\xi$,

$$
\alpha(\mathbf{k})=\alpha_{0}(\mathbf{k})+\xi \alpha_{1}(\mathbf{k}), \quad \beta(\mathbf{k})=\beta_{0}(\mathbf{k})+\xi \beta_{1}(\mathbf{k}) .
$$

The functions $\alpha_{0}, \alpha_{1}, \beta_{0}$ and $\beta_{1}$ can be computed at any moment $\eta$ by numerical solution of equations (14)-(19) with the initial conditions (22). The equations are accurate in the tight-coupling regime until some moment before the time of recombination $\eta_{r}$ defined below, and in the limit of sharp transition from opacity to transparency we can use them up to the moment $\eta_{r}$. The behavior of the functions $\alpha_{0}, \alpha_{1}, \beta_{0}$ and $\beta_{1}$ at that moment is shown in fig. 1. In the calculations we have used the values $\zeta_{s}=10^{-9}$ and $\zeta_{\text {in }}=10^{-5}$, and for the viscosity coefficient entering equation (15) we have used the formula

$$
\mathcal{N}=\frac{4}{15} \frac{\rho^{\gamma}}{\sigma_{T} n_{e} X_{e}}
$$

where $\rho^{\gamma}$ is the photon energy density, $\sigma_{T}$ is Thomson cross section, $n_{e}$ is the total number density of electrons and $X_{e}$ is the ionization fraction. For the redshift $z$ up to 1200 we have approximated the ionization fraction by the formula (3.202) in [17, and for $z$ over 1200 we have used an exponential function converging to 1 for $z$ going to infinity and matching the low-redshift function together with its derivative at $z=1200$. Note that the formula (33) has been derived without taking into account the polarization of photons. In a more accurate 

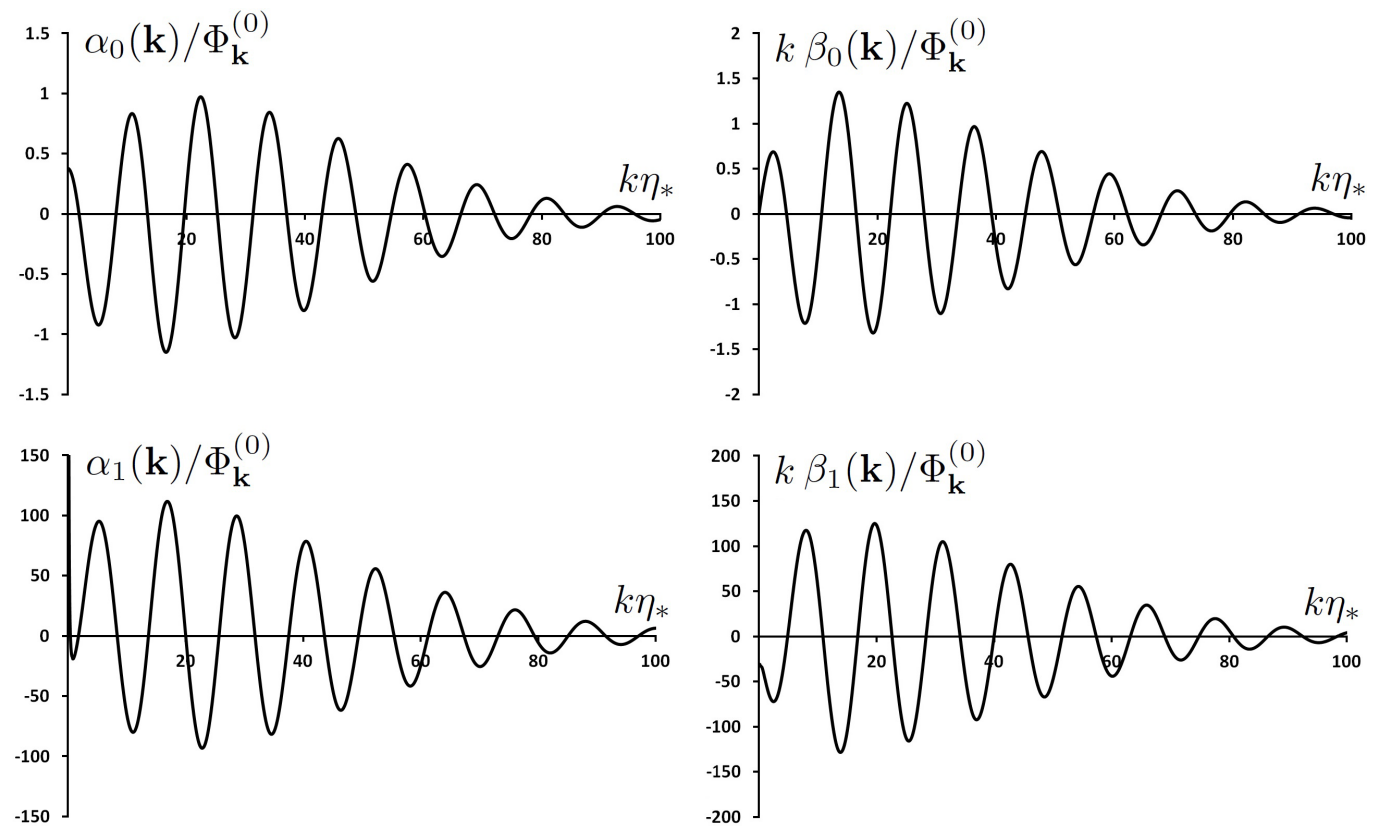

Fig. 1: Functions $\alpha_{0}, \alpha_{1}, k \beta_{0}$ and $k \beta_{1}$ at the moment $\eta=\eta_{r}$, divided by the primordial value of the Newtonian potential $\Phi_{\mathbf{k}}^{(0)}$. The ratios depend only on the comoving wavenumber $k$ and not on the direction of the vector $\mathbf{k}$, because the equations governing the evolution of perturbations are isotropic.

formula, the factor $4 / 15$ is replaced by $16 / 45$, see $\S 6.4$ in [16]; however, the results presented in the next section are not affected significantly by this correction.

Note that $\alpha_{1}$ diverges in the long-wavelength limit being proportional to $k^{-2}$ for small $k$. This divergence occurs due to the gauge transformation in (23), while variables in propertime comoving gauge have no divergences so that the perturbation theory remains applicable. This is the conceptual reason for using proper-time comoving gauge in our problem.

Due to the finite duration of recombination, the observer sees photons which scattered for the last time at different moments. This is called finite thickness effect. The probability that the photon was scattered within the given conformal time interval and then avoided further scattering until the conformal time $\eta_{0}$, is given by the probability density function

$$
\mathcal{P}(\eta)=\frac{d \mu(\eta)}{d \eta} e^{-\mu(\eta)}, \quad \mu(\eta)=\int_{\eta}^{\eta_{0}} \frac{a}{\tau} d \eta
$$

where $\tau$ is the mean free time for Thomson scattering, which varies with time, and $\mu$ is the optical depth. The function $\mathcal{P}$ is called visibility function. Due to the finite thickness effect, the overall fluctuations of the CMB temperature as seen by an observer at the present moment can be written as

$$
\frac{\delta T}{T}\left(\eta_{0}, \mathbf{l}\right)=\int \frac{\delta T}{T}\left(\eta_{0}, \mathbf{l} ; \eta_{l}\right) \mathcal{P}\left(\eta_{l}\right) d \eta_{l} .
$$

Since the visibility function can be approximated by a Gaussian function (see (7.2.25) in [16] or (9.55) in [17]), the observed relative anisotropies in the CMB temperature can be written as

$$
\frac{\delta T}{T}\left(\eta_{0}, \mathbf{l}\right)=\int \frac{d^{3} k}{(2 \pi)^{3 / 2}}\left(\alpha(\mathbf{k})+\beta(\mathbf{k}) \partial_{0}\right)_{\eta_{r}} e^{i \mathbf{k} \cdot \mathbf{l}\left(\eta_{r}-\eta_{0}\right)} e^{-\Sigma k^{2}}
$$


where the conformal time of recombination $\eta_{r}$ is the time when the visibility function is maximal and the function $\Sigma$ is defined as $\Sigma=\left(6 \kappa^{2} \mathcal{H}\left(\eta_{r}\right)^{2}\right)^{-1}, \kappa$ being the ratio of the ionization energy in the $2 \mathrm{~S}$ state of the hydrogen atom to the energy $k_{B} T_{r}$ corresponding to the temperature of recombination $T_{r}$ (see $\S 3.6 .3$ in [17]).

\section{Angular power spectrum}

Relative anisotropies in the CMB temperature can be expanded into spherical harmonics, with the coefficients that can be written as scalar products of the anisotropy with the corresponding spherical harmonic,

$$
\frac{\delta T}{T}\left(\eta_{0}, \mathbf{l}\right)=\sum_{l m} a_{l m} Y_{l m}(\mathbf{l}), \quad a_{l m}=\int d^{2} \mathbf{l} \frac{\delta T}{T}\left(\eta_{0}, \mathbf{l}\right) Y_{l m}^{*}(\mathbf{l}) .
$$

Since the CMB anisotropies are random, the coefficients $a_{l m}$ are random as well, and in order to compare the theory with the observational data, correlation functions must be introduced. The two-point correlation function is defined as $C(\theta)=\left\langle\delta T\left(\eta_{0}, \mathbf{l}_{1}\right) \delta T\left(\eta_{0}, \mathbf{l}_{2}\right) / T^{2}\right\rangle$, where $\theta$ is the angle between $\mathbf{l}_{1}$ and $\mathbf{l}_{2}$ and the angular brackets denote averaging over observer's positions. This function can be rewritten as a sum over multipole moments

$$
C(\theta)=\frac{1}{4 \pi} \sum_{l}(2 l+1) C_{l} P_{l}(\cos \theta),
$$

where $C_{l}=\left\langle\left|a_{l m}\right|^{2}\right\rangle$ are the coefficients of the angular power spectrum of CMB and $P_{l}$ are Legendre polynomials. The mean values $\left\langle\left|a_{l m}\right|^{2}\right\rangle$ are independent on $m$ because there is no preferred direction in the universe, but for the given observer there always exists statistical randomness in the CMB anisotropies, so that the observed values of $\left|a_{l m}\right|^{2}$ with the given multipole moment $l$ are not the same for all $m$. The best estimate of the coefficients of angular power spectrum is then given by averaging

$$
C_{l}=\frac{1}{2 l+1} \sum_{m=-l}^{l}\left|a_{l m}\right|^{2}
$$

with an unavoidable error known as the cosmic variance,

$$
\frac{\Delta C_{l}}{C_{l}}=\sqrt{\frac{2}{2 l+1}} .
$$

Using (36) and (37) we find

$$
C_{l}=\frac{2}{\pi} \int_{0}^{\infty} \tau_{l}(k)^{2}(2 \pi)^{3} \mathcal{P}_{\Phi}(k) k^{2} d k
$$

where the functions $\tau_{l}(k)$ are defined as

$$
\tau_{l}(k)=\left[\frac{\alpha(\mathbf{k})}{\Phi_{\mathbf{k}}^{(0)}} j_{l}\left(k\left(\eta_{0}-\eta_{r}\right)\right)+\frac{k \beta(\mathbf{k})}{\Phi_{\mathbf{k}}^{(0)}} j_{l}^{\prime}\left(k\left(\eta_{0}-\eta_{r}\right)\right)\right] e^{-\Sigma k^{2}},
$$

$j_{l}$ being the spherical Bessel functions of the first kind, and the function $\mathcal{P}_{\Phi}(k)$, known as power spectrum, is defined by

$$
\left\langle\Phi_{\mathbf{k}}^{(0)} \Phi_{\mathbf{k}^{\prime}}^{(0)}\right\rangle=(2 \pi)^{3} \mathcal{P}_{\Phi}(k) \delta^{(3)}\left(\mathbf{k}+\mathbf{k}^{\prime}\right) .
$$


The functions $\tau_{l}(k)$ depend only on the comoving wavenumber $k$ because they are solutions to the equations governing the evolution of perturbations, which are isotropic, with the primordial Newtonian potential $\Phi_{\mathbf{k}}^{(0)}$ appearing in the initial conditions replaced by 1 . The power spectrum is usually written as $\mathcal{P}_{\Phi}(k) \propto k^{n_{s}-4}$, where $n_{s}$ is the spectral index given by the inflationary scenario describing the formation of perturbations. In our calculations we have used the Planck value of spectral index $n_{s}=0.96$, which corresponds to the primordial spectrum slightly tilted downwards.

Define the variance of the coefficients of angular power spectrum due to the presence of radiation-like solid as

$$
\frac{\Delta_{\xi} C_{l}}{C_{l}}=\frac{C_{l}(\xi)-C_{l}}{C_{l}}
$$

where $C_{l}$ and $C_{l}(\xi)$ are the coefficients of angular power spectrum in a universe without the radiation-like solid and with it respectively. The values of $\Delta_{\xi} C_{l} / C_{l}$ are compared with the cosmic variance for the Planck values of cosmological parameters in fig. 2 and 3 . The

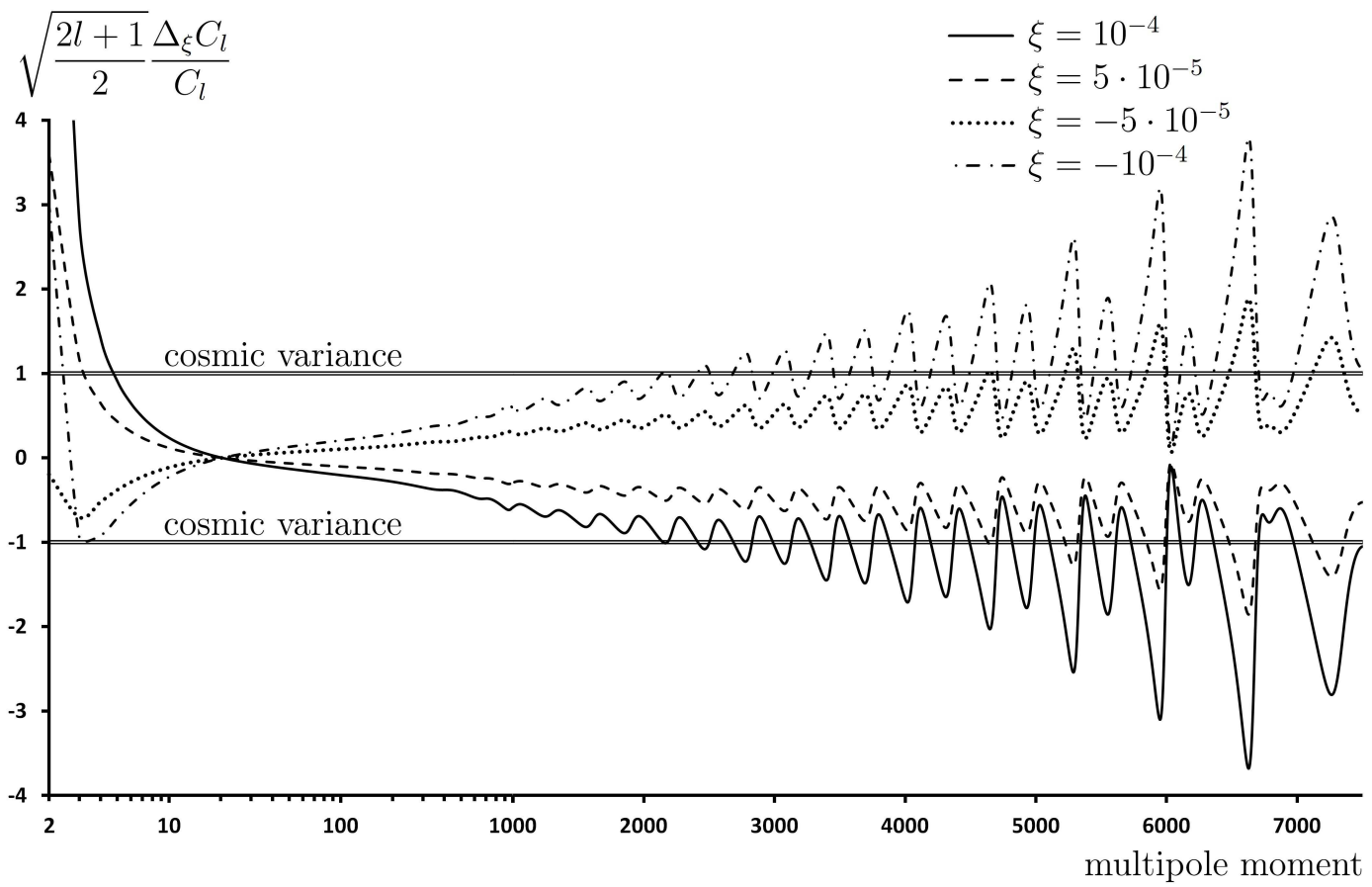

Fig. 2: The ratio of the variance of the coefficients of angular power spectrum due to the presence of radiation-like solid to the cosmic variance, depicted for given values of the shear modulus parameter $\xi$. On the horizontal axis we have combined logarithmic scale (for $l$ up to $1000)$ with ordinary scale ( $l$ over 1000$)$. The variance due to the presence of the radiation-like solid surpasses the cosmic variance for very high multipole moments as well as for very low ones if the integrated Sachs-Wolfe effect is not considered. With the integrated Sachs-Wolfe effect included, the effect of radiation-like solid for low multipole moments is diminished, so that the curves get under the cosmic variance for low multipole moments while not changing for high ones.

integrated Sachs-Wolfe effect was not included into calculations since its contribution for high multipole moments is negligible. For very high multipole moments, we have simplified the calculation of the integral (41) by using the approximate formula (9.72) in [17]. Note that in order to calculate $C_{l}$ up to $l=7500$, one has to know the functions $\alpha(\mathbf{k})$ and $\beta(\mathbf{k})$ not just up to $k \eta_{*}=100$ as in fig. 1 , but at least up to $k \eta_{*} \sim 150$. 


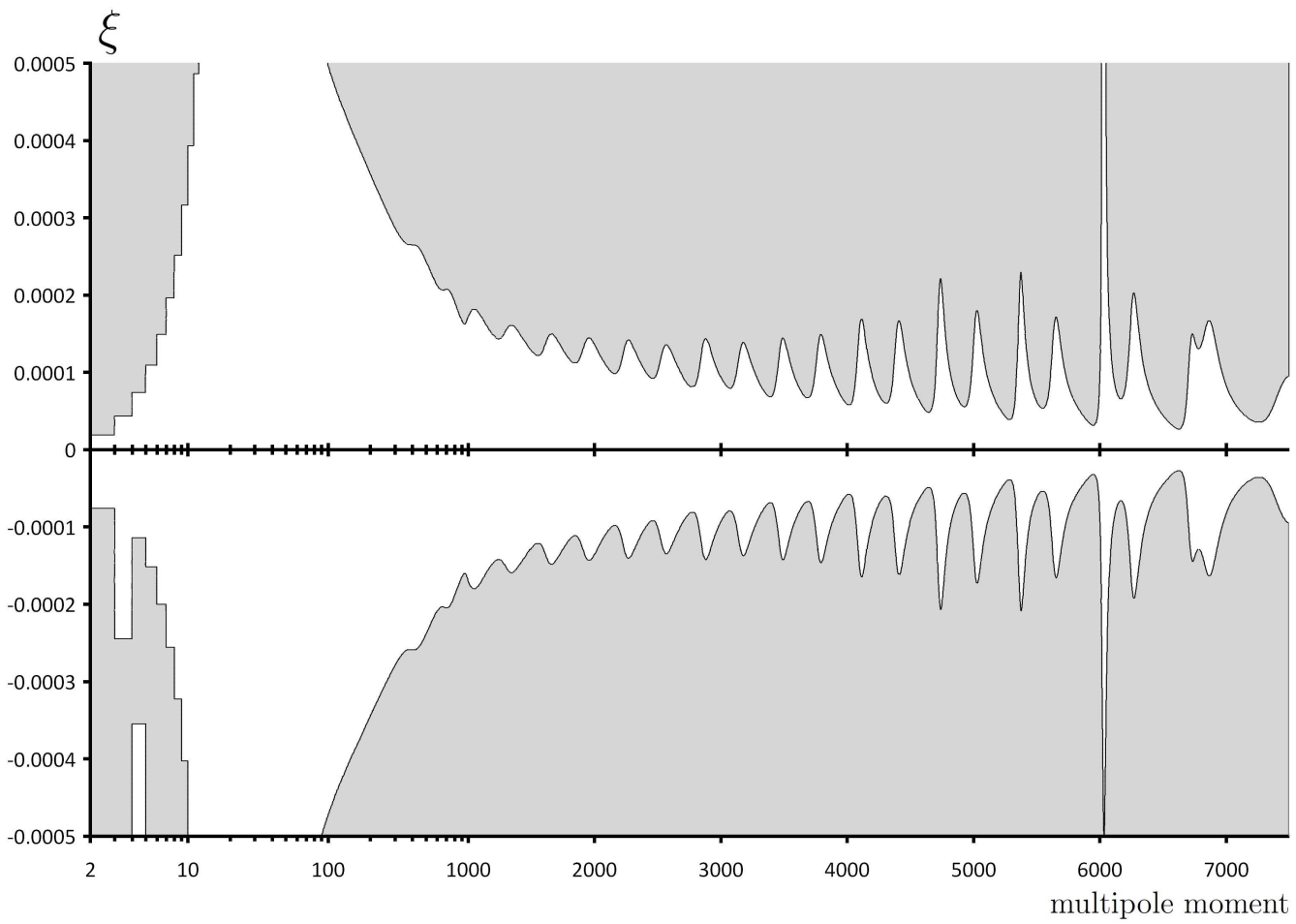

Fig. 3: The values of the parameter $\xi$ for which the variance due to the presence of the radiation-like solid exceeds the cosmic variance (grey area). For the horizontal axis we have combined logarithmic scale (for $l$ up to 1000) with ordinary scale ( $l$ over 1000).

The coefficient $C_{2}(\xi)$ diverges due to the contribution of long-wavelength part of the function $\tau_{2}(k)$ to the integral (41). However, because of finite duration of inflation the wavelengths of quantum fluctuations did not increase to infinity, hence the integral (41) is to be computed with nonzero lower limit $k_{\text {min }}$; then, no divergence occurs. The value of $k_{\min }$ can be estimated as $k_{\text {min }} \eta_{0} \sim e^{N_{\min }-N}$, where $N$ is the number of $e$-folds during inflation and $N_{\min }$ is the minimal value of $N$ needed to homogenize the universe on the scale of the present-day Hubble radius. We have used $k_{m i n} \eta_{*}=0.01\left(k_{m i n} \eta_{0} \doteq 0.5\right)$, which affects the angular power spectrum only for the lowest multipole moments.

An explicit calculation shows that the enhancement of viscosity due to the effects of polarization of photons, mentioned after equation (33), while affecting the coefficients $C_{l}(\xi)$ and $C_{l}$ of the angular power spectrum for high multipole moments significantly, changes the ratio $\Delta_{\xi} C_{l} / C_{l}$ only slightly. The reason is that for given multipole moment the coefficients $C_{l}(\xi)$ and $C_{l}$ are changed by similar factors. The same is true about the finite thickness effect, and presumably also about the free-streaming of photons and reionization. We thus believe that our results for high multipole moments are not qualitatively different from the results which one would obtain with the use of full Boltzmann equation, in the sense that for $|\xi| \gtrsim 10^{-4}$ the absolute value of $\sqrt{(2 l+1) / 2} \Delta_{\xi} C_{l} / C_{l}$ would still exceed 1 for multipole moments starting from a value between 2000 and 3000 depending on $\xi$, equal approximately to that obtained in our calculation.

The presence of radiation-like solid can be confirmed by observations only if $\left|\Delta_{\xi} C_{l} / C_{l}\right|$ $>\left|\Delta C_{l} / C_{l}\right|$. As seen from fig. 2 and 3 , this holds for very high $l$, even if $|\xi|$ is small enough so that inverse inequality is valid for low $l$. To verify that the latter is true one can make use of the result of [15] that for low $l$ the integrated Sachs-Wolfe effect suppresses the effect of radiation-like solid on $C_{l}$ by a factor of about 50 . 


\section{Conclusion}

We have applied perturbation theory in the proper-time comoving gauge to a universe which contains, in addition to radiation, baryonic matter and CDM, a radiation-like solid with the pressure to energy density ratio $w=1 / 3$ and constant shear modulus to energy density ratio $\xi$. The presence of such solid does not change the evolution of the unperturbed universe.

In order to obtain results also for short-wavelength perturbations, we had to extend the theory developed in [15] and suppose that the CDM and neutrinos were not coupled to the baryon-radiation plasma. This produced a more complicated system of equations; however, as shown in the appendix, the long-wave solution in both theories is the same. This provided us with a simple check of the computed CMB anisotropies for very low angular moments, since we could compare them directly with the results of [15].

We have calculated how a radiation-like solid influences the CMB angular power spectrum and found that its presence has a significant effect on the angular power spectrum not only for very low multipole moments, as shown in [15], but also for very high ones. It turns out that the effect of the solid is suppressed with the increasing multipole moment less than the error of the coefficients of angular power spectrum coming from the fact that we observe the universe just from one place. The observational error caused by the dust in our Galaxy decreases with multipole moment as well, therefore future observations of the CMB anisotropies with high enough resolution could confirm or refute the presence of the radiation-like solid in our universe with greater certainty than it is possible today.

For $|\xi| \sim 10^{-4}$, the effect is observable for $l \gtrsim 3000$, which is the limit of Planck satellite resolution. At the same time, according to [15], the large-angle anisotropies are not affected significantly for such values of $\xi$ if one considers also the integrated Sachs-Wolfe effect. We have not included this effect into our work because it affects the angular power spectrum significantly only for low multipole moments; however, if we take it into account we find that our model is in agreement with current observations for the values of the shear modulus parameter $\xi$ close enough to zero, while causing effects not predicted by the standard theory which are beyond the reach of current observations.

We have considered also negative $\xi$, which leads to instability of vector perturbations [14, but the theory may still be relevant since such perturbations are not created in standard inflationary models. We have not included free streaming of photons into the theory, which however should not change the results qualitatively.

Acknowledgement. The work was supported by the grant VEGA 1/0985/16.

\section{A Long-wave solution}

Equations of motion (14)-19) can be solved analytically in the long-wavelength limit $(s \ll 1)$. In order to do so it is useful to rewrite them in terms of new variables $\Delta_{E}^{d}=\hat{\delta}^{d}-\mathcal{E}$, 
$\Delta_{B}^{d}=\Theta-\tilde{\mathcal{B}}, \Delta_{E}^{\nu}=\hat{\delta}^{\nu}-(4 / 3) \mathcal{E}$ and $\Delta_{B}^{\nu}=\Sigma-\tilde{\mathcal{B}}$. The resulting equations are

$$
\begin{aligned}
\mathcal{E}^{\prime} & =-s^{2} \tilde{\mathcal{B}}-\alpha \tilde{\mathcal{H}} \epsilon-\alpha_{d} \tilde{\mathcal{H}} \mathcal{D}_{E}^{d}-\alpha_{\nu} \tilde{\mathcal{H}} \mathcal{D}_{E}^{\nu}, \\
\tilde{\mathcal{B}}^{\prime} & =c_{s 0}^{2} \epsilon+\Xi \mathcal{E}^{\prime}+(\alpha-1) \tilde{\mathcal{H}} \tilde{\mathcal{B}}+\alpha_{d} \tilde{\mathcal{H}} \Delta_{B}^{d}+\alpha_{\nu} \tilde{\mathcal{H}} \Delta_{B}^{\nu}+3 \xi c_{s 0}^{2} \mathcal{E}, \\
\Delta_{B}^{d^{\prime}} & =-c_{s 0}^{2} \epsilon-\tilde{\mathcal{H}} \Delta_{B}^{d}-3 \xi c_{s 0}^{2} \mathcal{E}, \\
\Delta_{E}^{d^{\prime}} & =-s^{2} \Delta_{B}^{d}, \\
\Delta_{B}^{\nu \prime} & =-\left(c_{s 0}^{2}-\frac{1}{3}\right) \epsilon+\frac{1}{4} \Delta_{E}^{\nu}-3 \xi c_{s 0}^{2} \mathcal{E}, \\
\Delta_{E}^{\nu \prime} & =-\frac{4}{3} s^{2} \Delta_{B}^{\nu},
\end{aligned}
$$

where $\alpha=3 \rho_{+} /(2 \rho), \epsilon=3 \tilde{\mathcal{H}} \tilde{\mathcal{B}}+\mathcal{E}, \mathcal{D}_{E}^{d}=3 \tilde{\mathcal{H}} \Delta_{B}^{d}+\Delta_{E}^{d}$ and $\mathcal{D}_{E}^{\nu}=3 \tilde{\mathcal{H}} \Delta_{B}^{\nu}+(3 / 4) \Delta_{E}^{\nu}$. We will consider this system of differential equations after the solidification time $\zeta_{s}$, when the parameter $\xi$ is nonzero. Assuming the solidification time to be close to zero, $\zeta_{s} \ll 1$, the initial conditions are $\Delta_{E}^{d}=0, \Delta_{B}^{d}=0, \Delta_{E}^{\nu}=0, \Delta_{B}^{\nu}=0, \mathcal{E}=-(9 / 2) \Phi^{(0)}$ and $\mathcal{B}=0$. From now on we set $\Phi^{(0)}=1$.

Our goal is to determine the potentials $\Phi$ and $\Psi$ and the photon energy density contrast $\delta_{\gamma}$, which are needed to calculate the coefficients of the angular power spectrum by using the integral (41). From the first equation in (23) it follows

$$
\mathcal{E}^{\prime}=s^{2}\left(\tilde{\mathcal{H}}^{-1} \Psi-\tilde{\mathcal{B}}\right),
$$

therefore in the long-wavelength limit the function $\mathcal{E}$ can be approximated by a constant. The initial condition for $\mathcal{E}$ then yields

$$
\mathcal{E}=-\frac{9}{2}
$$

Similarly, from equations (A-4) and (A-6) and the corresponding initial conditions we get

$$
\Delta_{E}^{d}=0, \quad \Delta_{E}^{\nu}=0 .
$$

However, while the deviation of $\mathcal{E}$ from the value (A-8) is of order $s^{2}$, the deviations of $\Delta_{E}^{d}$ and $\Delta_{E}^{\nu}$ from zero are of order $s^{4}$. We will use these results in the following considerations.

The potential $\Psi$ can be rewritten with the help of (A-1) and the first equation in (23) as

$$
\Psi=\varphi-s^{-2} \tilde{\mathcal{H}}^{2}\left(\alpha_{d} \mathcal{D}_{E}^{d}+\alpha_{\nu} \mathcal{D}_{E}^{\nu}\right)
$$

where $\varphi=-s^{-2} \tilde{\mathcal{H}}^{2} \alpha \epsilon$. Denote $X=a / a_{e q}=\zeta(\zeta+2)$. Using equations of motion in the zeroth and first order of the perturbation theory and solutions (A-8) and (A-9), we can write the derivative of $\varphi$ with respect to $\zeta$ as

$$
\varphi^{\prime}=\left(\frac{f^{\prime}}{f}+\frac{\hat{f}^{\prime}}{\hat{f}}\right) \varphi+C+216 \xi s^{-2} f \hat{f},
$$

where $f=(\zeta+1) X^{-3}, \hat{f}=(4+3 X) /\left(4+3 N_{0} x_{b} X\right), N_{0}$ being the ratio of energy density of radiation including neutrinos to photon energy density and $x_{b}$ being the ratio of baryon energy density to the energy density of baryonic matter and CDM, and $C=\tilde{\mathcal{H}}^{2} \alpha \mathcal{B}$. The function $C$ can be written as

$$
C=\tilde{\mathcal{H}}^{2} \alpha \tilde{\mathcal{B}}_{\xi=0}+\mathcal{C}(\xi)
$$

where $\tilde{\mathcal{B}}_{\xi=0}$ denotes the function $\tilde{\mathcal{B}}$ in a universe with no solid (solution of equations for perturbations in which the shear modulus parameter $\xi$ is set to zero) and $\mathcal{C}$ is a function of 
first order in $\xi$. Obviously, the function $\mathcal{C}$ is much smaller than the third term in A-11 in the long-wavelength limit, so that the function $C$ can be computed assuming that $\xi$ is zero.

With $\mathcal{E}$ given by (A-8) and $s \ll 1$, equation (A-1) reduces to $\alpha \epsilon+\alpha_{d} \mathcal{D}_{E}^{d}+\alpha_{\nu} \mathcal{D}_{E}^{\nu}=0$. Furthermore, by inserting from (A-9) into the definitions of $\mathcal{D}_{E}^{d}$ and $\mathcal{D}_{E}^{\nu}$ we get

$$
\mathcal{D}_{E}^{d}=3 \tilde{\mathcal{H}} \Delta_{B}^{d}, \quad \mathcal{D}_{E}^{\nu}=3 \tilde{\mathcal{H}} \Delta_{B}^{\nu} .
$$

Hence, if $\xi$ is zero, $\epsilon$ can be expressed as a linear combination of $\Delta_{B}^{d}$ and $\Delta_{B}^{\nu}$ (with the coefficients depending on the time $\zeta$ ) and, therefore, equations (A-3) and (A-5) can be reduced to a system of linear differential equations for $\Delta_{B}^{d}$ and $\Delta_{B}^{\nu}$. The only solution of this system satisfying the initial conditions is $\Delta_{B}^{d}=0$ and $\Delta_{B}^{\nu}=0$, which implies that $\epsilon$ must be zero as well. Therefore, using the definition of $\epsilon$ and $(\hat{A-8})$, we get $\tilde{\mathcal{B}}_{\xi=0}=3 /(2 \tilde{\mathcal{H}})$, and by inserting this into the expression for the function $C$ we find that $C$ can be approximated as

$$
C=\frac{3}{2} \tilde{\mathcal{H}} \alpha=\frac{3}{2} \frac{4+3 X}{X(\zeta+1)}
$$

After determining the unknown function on the right hand side of the differential equation (A-11), we can find $\varphi$ by solving this equation. With the substitution $\varphi=f \hat{f} F$, equation (A-11) yields

$$
F^{\prime}=\frac{C}{f \hat{f}}+216 \xi s^{-2}
$$

and by a straightforward integration we obtain

$$
F=6 \zeta^{3}\left[\varepsilon \zeta\left(\frac{1}{5} \zeta+1\right)+\frac{1}{3}+\varepsilon+(1-\varepsilon) \frac{1}{\zeta+1}\right]+216 \xi s^{-2} \zeta
$$

where $\varepsilon=(3 / 4) N_{0} x_{b}$. In order that the contribution of the solid to the perturbations is comparable to or less than the contribution of the remaining components of matter, we require that $\xi s^{-2} \lesssim 1$.

To compute $\Psi$ we need to know, in addition to the function $\phi$, the functions $\mathcal{D}_{E}^{d}$ and $\hat{\tilde{\Delta}}_{E}$ up to the order $s^{2}$. However, as mentioned before, the functions $\Delta_{E}^{d}$ and $\tilde{\Delta}_{E}^{d}$ are of order $s^{4}$; thus, it suffices to know the functions $\Delta_{B}^{d}$ and $\tilde{\Delta}_{B}^{d}$ up to the order $s^{2}$. These functions are given by equations (A-3) and , which can be rewritten as

$$
\Delta_{B}^{d}{ }^{\prime}=s^{2} \frac{D}{X} f \hat{f} F-\tilde{\mathcal{H}} \Delta_{B}^{d}+\frac{27}{2} \xi c_{s 0}^{2},
$$

and

$$
\Delta_{B}^{\nu \prime}=-s^{2} \varepsilon D f \hat{f} F+\frac{27}{2} \xi c_{s 0}^{2},
$$

where $D=X c_{s 0}^{2} /\left(\tilde{\mathcal{H}}^{2} \alpha\right)=(3 / 2) X^{4} /(4+3 X)^{2}$ and the sound speed squared can be written as a function of time as $c_{s 0}^{2}=1 /[3(1+\varepsilon X)]$. The latter equation integrates immediately and the former equation can be integrated as well after passing from $\Delta_{B}^{d}$ to $X \Delta_{B}^{d}$.

For the potential $\Psi$ we have

$$
\Psi=f \hat{f} F-s^{-2}\left(E_{d} \Delta_{B}^{d}+E_{\nu} \Delta_{B}^{\nu}\right)
$$

where $E_{d}=3 \tilde{\mathcal{H}}^{3} \alpha_{d}=36 x_{d} f X$ and $E_{\nu}=3 \tilde{\mathcal{H}}^{3} \alpha_{\nu}=48 \nu f$, with CDM energy density to energy density of CDM and baryonic matter ratio denoted by $x_{d}$ and neutrino energy density to the energy density of all kinds of radiation ratio denoted by $\nu, x_{d}=1-x_{b}$ and $\nu=$ $1-N_{0}^{-1}$. Inserting for $F$ the expression (A-16) and for $\Delta_{B}^{d}$ and $\tilde{\Delta}_{B}^{d}$ the expressions obtained 
by integrating equations (A-17) and (A-18), after some algebra we arrive at the formula derived in [15] for a universe in which all kinds of matter are coupled to each other. The formula reads

$$
\Psi=\frac{3}{2} f \zeta^{3}\left(\frac{3}{5} \zeta^{2}+3 \zeta+\frac{13}{3}+\frac{1}{\zeta+1}\right)+216 \tilde{\xi} s^{-2} f \zeta
$$

where $\tilde{\xi}=\mu / \rho^{s r}, \rho^{s r}=N_{0} \rho^{s \gamma}$ being the total energy density of the solid and radiation (photons and neutrinos).

Having determined $\Psi$, we can compute the Newtonian potential $\Phi$ from the second equation in (23). We obtain immediately the same expression as in 15,

$$
\Phi=\Psi-108 \tilde{\xi} s^{-2} \frac{1}{X^{2}} .
$$

Finally, from equation (25) we find that the photon energy density contrast is $\delta_{\gamma}=4 \Psi-6$. As a result, the function $\alpha(\mathbf{k})$ can be written in terms of $\Phi$ and $\Psi$ as $\alpha(\mathbf{k})=\Phi+\Psi-3 / 2$.

In the calculation of the coefficients of angular power spectrum we need the value of the function $\alpha(\mathbf{k})$ at the time of recombination $\zeta=\zeta_{r}$. For cosmological parameters which are obtained from the data assembled by the satellite Planck we have $\zeta_{r}=1.062$, which yields $\alpha_{r}(\mathbf{k})=0.371+17.3 \tilde{\xi} s^{-2}$.

To find the contribution of Silk damping to the temperature fluctuations we need to extend the expression for $\Phi$ by the viscosity term which has been omitted in the second equation (23). (Viscosity term in the equations for perturbations was kept, since it plays an important role for wavenumbers which are not small.) Viscosity contributes to $\Phi$ and $\Psi$ by terms of order $k^{0}$ or $\xi k^{-2}$ so that it is in principle relevant in the long-wavelength limit, however, because the viscosity coefficient is small, the Silk damping can be neglected in the considered limit. Since the function $k \beta(\mathbf{k})$ is zero in this limit, the Doppler effect can be neglected as well. On the other hand, contribution of integrated Sachs-Wolfe effect cannot be neglected for small multipole moments. To calculate it one can use relations (64) in [15], with additional terms originating in the decoupling of CDM and neutrinos. 


\section{References}

[1] N. Bucher, D. N. Spergel, Phys. Rev. D60, 043505 (1999)

[2] R. A. Battye, N. Bucher, D. Spergel, astro-ph/9908047

[3] A. Leite, C. Martins, Phys. Rev. D84, 103523 (2011)

[4] R. A. Battye, A. Moss, Phys. Rev. D74, 041301 (2006)

[5] R. A. Battye, A. Moss, Phys. Rev. D80, 023531 (2009)

[6] R. A. Battye, A. Moss, Phys. Rev. D76, 023005 (2007)

[7] R. A. Battye, J. A. Pearson, Phys. Rev. D88, 084003 (2013)

[8] S. Kumar, A. Nautiyal, A. A. Sen, Eur. Phys. J. C73, 2562 (2013)

[9] A. Gruzinov, Phys. Rev. D70, 063518 (2004)

[10] S. Endlich, A. Nicolis, J. Wang, JCAP 1310, 011 (2013)

[11] M. Akhshik, JCAP 1505, 043 (2015)

[12] N. Bartolo, S. Matarrese, M. Peloso and A. Ricciardone, JCAP 1308, 022 (2013)

[13] M. Sitwell, K. Sigurdson, Phys. Rev. D89, 123509 (2014)

[14] V. Balek, M. Škovran, arXiv:1401.7004 [gr-qc]

[15] V. Balek, M. Škovran, Class. Quant. Grav. 32, 015015 (2015)

[16] S. Weinberg: Cosmology, Oxford University Press (2008)

[17] V. Mukhanov: Physical Foundations of Cosmology, CUP, Cambridge (2005)

[18] V. Polák, V. Balek, Class. Quant. Grav. 25, 045007 (2008)

[19] A. R. Liddle, D. H. Lyth: Cosmological Inflation and Large-Scale Structure, CUP, Cambridge (2000) 